\title{
The role of grain boundary structure and crystal orientation on crack growth asymmetry in aluminum
}

\author{
I. Adlakha ${ }^{1}$, M.A. Tschopp ${ }^{2}$, and K.N. Solanki ${ }^{1 *}$ \\ ${ }^{1}$ School for Engineering of Matter, Transport, and Energy; Arizona State University; Tempe, AZ \\ 85287, USA \\ ${ }^{2}$ U.S. Army Research Laboratory, Aberdeen Proving Ground, MD 21005, USA \\ *(480)965-1869; (480)727-9321 (fax), E-mail: kiran.solanki@asu.edu, (Corresponding author)
}

\begin{abstract}
Atomistic simulations have shown that the grain boundary (GB) structure affects a number of physical, mechanical, thermal, and chemical properties, which can have a profound effect on macroscopic properties of polycrystalline materials. The research objective herein is to use atomistic simulations to explore the role that GB structure and the adjacent crystallographic orientations have on the directional asymmetry of an intergranular crack (i.e. cleavage behavior is favored along one direction, while ductile behavior along the other direction of the interface) for aluminum grain boundaries. Simulation results from seven $<110>$ symmetric tilt grain boundaries (STGBs) show that the GB structure and the associated free volume directly influence the stress-strain response, crack growth rate, and crack tip plasticity mechanisms for middle-tension $(\mathrm{M}(\mathrm{T}))$ crack propagation specimens. In particular, the structural units present within the GB promote whether a dislocation or twinning-based mechanism operates at the crack tip during intergranular fracture along certain GBs (e.g., the 'E' structural unit promotes twinning at the crack tip in Al). Furthermore, the crystallography of the adjacent grains, and therefore the available slip planes, can significantly affect the crack growth rates in both directions of the crack - this creates a strong directional asymmetry in the crack growth rate in the $\Sigma 11$ (113) and the $\Sigma 27$ (552) STGBs. Upon comparing these results with the theoretical Rice criterion, it was found that certain GBs in this study ( $\Sigma 9$ (221), $\Sigma 11$ (332) and $\Sigma 33$ (441)) show an absence of directional asymmetry in the observed crack growth behavior, in conflict with the Rice criterion. The significance of the present research is that it provides a physical basis for the role of GB character and crystallographic orientation on intergranular crack tip deformation behavior.
\end{abstract}

Keywords: Grain boundary; Twinning; Dislocation; Fracture; Directional anisotropy 


\section{Introduction}

Atomistic modeling and simulation methods have dedicated considerable effort towards understanding the brittle versus ductile behavior of materials, including the dynamic instabilities that occur during fracture [1-11]. To understand this behavior, a number of studies have focused the deformation process that occurs at the crack tip, i.e., brittle (cleavage) versus ductile (nucleation of partial and full dislocations, and deformation twinning) behavior and dislocation burst in single crystal and bicrystal metals, in particular [6]. These simulations have clarified the effects of applied load orientation and slip planes on dislocation nucleation or twinning at/ahead of the crack tip, both in the single crystal and at the grain boundaries (GBs).

In general, atomistic simulations have helped in understanding that crack tip propagation is primarily governed by the dislocation process (emission versus cleavage) and in some metals, such as aluminum and copper, deformation may be accommodated through deformation twinning [10,12-14]. Deformation twinning, especially in aluminum, has been a subject of debate due to discrepancies in experimental and atomistic modeling results, regardless of the time and length scale issues [2,3,10,13]. For example, Tadmor and Hai [15] used a quasicontinuum method to suggest that deformation twins form ahead of the crack tip in single crystal

aluminum when $T=\lambda_{\text {crit }} \sqrt{\gamma_{u s} / \gamma_{u t}}>1$, where $\lambda_{\text {crit }}$ is the normalized critical load for the nucleation of a trailing partial dislocation, $\gamma_{u s}$ is the energy associated with unstable stacking fault energy, and $\gamma_{u t}$ is the unstable twinning partial nucleation energy. Farkas et al. [2] and others $[6,16,17]$ have used atomistic simulations to observe similar twinning behavior in Al. However, it has been well-established experimentally that Al does not twin except under certain loading conditions and at relative small time scales, primarily due to the high stacking fault energy of the material $[13,17,18]$. On the other hand, this discrepancy between simulations and experiments regarding deformation twinning does not occur in nanocrystalline (NC) metals (grain size $<\sim 100 \mathrm{~nm}$ ), as observed by Zhu et al. [12,19] and others [16,17,20-22], where deformation twinning is indeed one of the experimentally-observed deformation mechanisms, along with the GB rotation/sliding and dislocation glide [12,17,21]. This behavior may be partially attributed to GB interfaces and networks that contribute significantly to the properties of metals. Furthermore, onset of plastic deformation is complicated due to the dissociated and faceted structure present in GB interfaces [23,24]. Hence, the local structure and character of the GB was important for understanding interfacial crack mechanics. 
Research has shown that GB properties are affected by both the macroscopic degrees of freedom and the microscopic local structure [25-30]. The term GB character is often used to refer to the macroscopic GB degrees of freedom. The GB character refers to the five degrees of freedom associated with the misorientation between the crystallographic orientations of the two adjoining grains. Hence, GB character encompasses not only the misorientation angle, but also the GB plane as well as information pertaining to GB type (e.g., low angle versus high angle, $\Sigma$ value, etc.). In terms of microscopic local structure, the translations between adjoining grains are important, as is the localized dislocation structure of the boundary. Historically, many efforts have focused on developing a method to characterize GBs [31-36] and their influence on the physical properties of polycrystalline material (e.g. [37-39]). These models utilized dislocation arrays, disclinations, and coincident site lattice (CSL) to describe microscopic and macroscopic degrees of freedom of GBs. Based on identifying the favored GB for the corresponding GB systems, these methodologies [25,28,40-46] described the structural elements comprising symmetric tilt, asymmetric tilt, twist, and twin GBs. They determined that the favored GBs are entirely composed of unique structural units (SUs) that cannot be decomposed into other GB structures.

There have been several studies that examine the role of GB structural units on GB plasticity and deformation mechanisms. For instance, Sansoz and Molinari [47] used quasi-continuum simulations on $\mathrm{Al}$ and $\mathrm{Cu}$ bicrystals to show that deformation sliding was accommodated through atomic rearrangement of 'E' SUs in the GB structure for the $<110>$ symmetric tilt grain boundary (STGB) system. Additionally, Spearot et al. $[23,48]$ have used molecular dynamics (MD) simulations at $10 \mathrm{~K}$ and $300 \mathrm{~K}$ to examine how GB structural units impacted the process of dislocation nucleation. However, the role of GB character on plastic events such as dislocation emission, twin formation, and atomic displacements at the interface with a preexisting crack has received less attention, especially in $\mathrm{Al}$ with varying types of GB SUs. In fact, in $\mathrm{Cu}$ and $\mathrm{Fe}$ bicrystals, it has been shown experimentally that there exists a strong directional dependence on the fracture behavior along the interface [49-54]. Moreover, the crack growth asymmetry is governed by the relative angle of the slip plane to the crack plane $(\theta)$ and the angle between normal to the crack tip and slip direction $(\phi)$ [55], as depicted in Figure 1a. The critical energy release rate $(\mathrm{G})$ required for a dislocation emission can be expressed as follows: 


$$
G_{\text {disl }}=8\left(\frac{1+(1-\vartheta) \tan ^{2} \phi}{(1+\cos \theta) \sin ^{2} \theta}\right) \gamma_{u s}
$$

where $\gamma_{u s}$ is the unstable stacking fault energy for the slip plane and $\vartheta$ is the Poisson's ratio. Furthermore, the Rice-Thompson criterion for dislocation nucleation versus cleavage failure (i.e., $\mathrm{G}_{\text {cleav }}=2 \gamma_{s}$ ) is given by

$$
\frac{\gamma_{s}}{\gamma_{u s}}>4\left(\frac{1+(1-\vartheta) \tan ^{2} \phi}{(1+\cos \theta) \sin ^{2} \theta}\right)
$$

where $\gamma_{s}$ is the surface energy required to create a free surface along the GB interface. Wang et al. $[49,51]$ used the Rice-Thompson condition to examine experimentally-observed directional crack growth behavior in $\mathrm{Fe}-\mathrm{Si}$ and $\mathrm{Cu}$ bicrystalline interfaces and found that the large variation in crack growth behavior was attributed to the relative orientation of slip system ahead of crack tips. Furthermore, the larger crack growth along certain directions was observed when the ratio of $\frac{\gamma_{s}}{\gamma_{u s}}$ was the lowest. For example, using the Rice criterion, Wang et al. [49,51] calculated the $G_{d i s l}$ for a dislocation nucleation from the $\Sigma 9(221) \mathrm{GB}$ in $\mathrm{Cu}$ and $G_{d i s l}$ was $\sim 5.5 \mathrm{~J} / \mathrm{m}^{2}$ and $\sim 2.4$ $\mathrm{J} / \mathrm{m}^{2}$ along the $-\mathrm{X}$ and $+\mathrm{X}$ directions, respectively. On the other hand, the critical energy for the cleavage $\left(G_{\text {clea }}\right)$ in the $\Sigma 9(221)$ GB interfaces was $\sim 2.5 \mathrm{~J} / \mathrm{m}^{2}$. These predictions for the $\Sigma 9$ (221) GB suggest that the fracture behavior changes from a ductile to brittle or vice versa, when the crack propagation direction along the interface changes, which was further corroborated with experimental findings. However, a complete understanding of the fundamental mechanisms associated with asymmetric deformation are inaccessible with experimental techniques, especially the atomic deformation ahead of the advancing crack, the role of GB character, and the resulting directional intergranular fracture. Thus, this presents significant challenges to developing unique state variables (e.g., free volume, GB energy and SU present along the grain boundary etc.) capable of being hierarchically transferred to higher-order models for predictive modeling.

Therefore, the objective of the present research is to understand the relationship between the local atomic structure and the crack mechanics at the GB interface. In this work, the directional anisotropy of an intergranular crack was studied for $<110>$ STGBs in Al to clarify the role of interface character on dislocation emission, formation of twins, and/or atomic 
displacement. The GB structural description corresponding to the minimum energy was characterized using the structural unit model (SUM) [25,42,56-58], and the initial free volume of each interface was calculated using a previously-described method [59]. The Rice criterion predictions for the directional behavior of incipient plastic event ahead of a crack tip in these GBs were compared against the observed crack tip event. A middle tension - M(T) - specimen was modeled using molecular dynamics with a constant tensile strain rate applied normal to the GB plane at $300 \mathrm{~K}$. The tensile stress-strain response of these $\mathrm{M}(\mathrm{T}) \mathrm{GB}$ specimens were analyzed to correlate the mechanical response to the GB structure. Subsequently, the crack tip plasticity and the directional crack tip response of these GB interfaces were examined. The incipient crack tip event observed deviates from the Rice criterion predictions in certain interfaces (e.g., $\Sigma 9$ (221), $\Sigma 11$ (332), and $\Sigma 33$ (441) GBs). Furthermore, the crack growth during the fracture process was calculated and a noticeable variation in crack growth was observed for the $+\mathrm{X}$ direction and $-\mathrm{X}$ direction (from the center of the specimen).

This paper is organized as follows. Section 2 briefly summarizes the simulation approach utilized herein. The results and discussion are presented in Section 3, including the atomic structure of GBs at $0 \mathrm{~K}$, the stress-strain responses of the interfaces, the crack growth along both $+\mathrm{X}$ and $-\mathrm{X}$ directions of each interface, and the atomic deformation for GB interfaces displaying interesting crack growth features. The simulation results reveal several interesting observations: (1) there was a strong asymmetry in crack growth due to the difference in crystallographic orientation of the $\{111\}$ slip planes on adjacent grains and the crack growth directions, (2) in some cases, the GB volume ahead of the crack tip underwent significant structural rearrangement, which subsequently influenced the crack propagation mechanism, (3) in most GBs, crack propagation was caused by alternating mechanisms of dislocation emission, followed by propagation of dislocation (blunting) and cleavage/crack advance, (4) the crack growth rates along the GB interface were strongly influenced by the initial free volume at the interface, i.e., faster crack growth was observed along interfaces with higher initial free volume, (5) the 'E' SU GBs show a distinct deformation behavior compared to other $<110>$ GB interfaces, primarily due to the ease of nucleating the intrinsic stacking fault (ISF) ahead of the crack tip, which further act as site for nucleation of partial dislocation, and (6) twin-deformation was observed ahead of the crack tip only in the GB interfaces with ' $E$ ' SU in the GB structural description. Finally, this work provides a framework for analyzing GB deformation when primarily 
influenced by the SUs. This approach can be used to inform non-local cohesive zone models of atomistic grain boundary details in an effort to capture the relevant interfacial physics for higherlength scale models.

\section{Computational Methods}

A parallel molecular dynamics code (large-scale atomic/molecular massively parallel simulator, LAMMPS [60]) with a semi-empirical embedded atom method (EAM) [61] potential was used to study the role of GB character on plastic events and the subsequent crack dynamic behavior in Al STGBs with $<110>$ tilt axis. In this work, we employed the EAM potential of Liu et al. [62] to describe the interactions between $\mathrm{Al}-\mathrm{Al}$ and for generating the impurity-free GB systems. The EAM potential was fitted to the experimental data and a set of ab-initio structural atomic forces calculated for large set of configurations. The surface energies, stacking fault energies (SFE) and many other physical properties calculated using Liu et al potential [62] were in good agreement with experimental findings and other EAM potentials (refer [62,63]). Furthermore, this potential has been shown to accurately reproduce the experimental solid-liquid binary phase diagram [64], quantify stable structure formations, the thermo-mechanical behavior of nanocrystalline materials [22], the mechanistic causes for the crossover from a dislocation to a grain boundary-based deformation mechanism with decreasing grain size; grain boundary structure and energies; and many other physical behaviors, including the stacking fault energy (SFE) in $\mathrm{Al}[64]$.

\subsection{Equilibrium grain boundary structures}

The GB structure and minimum energy were calculated using a bicrystal simulation cell with three-dimensional (3D) periodic boundary conditions consisting of two grains at $0 \mathrm{~K}$ as described by Rittner and Seidman [28]. The tilt axis was $<110>$ for the two grains, which were rotated such that their crystallographic orientations are symmetric about the grain boundary plane. The periodic boundaries were maintained with a separation distance of $25 \mathrm{~nm}$ between the boundaries. Several $0 \mathrm{~K}$ minimum energy GB structures were obtained through successive rigid body translations followed by an atom deletion technique and energy minimization using a nonlinear conjugate method [24,28-30,37-39]. In particular, grain boundary structures generated 
using this technique have been compared with experimental high-resolution transmission electron microscopy images [65-67].

Atomic rearrangement that occurs during minimization of the GB can be expressed as relative displacement of atomic planes normal to the GB [68]. This provides a microscopic measure for the excess volume or the free volume required to create the interface because the strain field associated with the interface at the atomistic scale decays as $e^{-z}$, where $\mathrm{z}$ is the distance normal to the GB plane. The free volume can be measured by finding the relative displacement of two parallel atomic planes nearest to the GB. This measure represents the local expansion undergone by crystals at the interface $\left(\Delta z^{*}\right)$.

\subsection{Molecular dynamic fracture simulation}

For dynamic fracture simulations, the minimized GB structures were modeled by prescribing periodic conditions along the $\mathrm{Y}$ and $\mathrm{Z}$ directions and a non-periodic condition along the $\mathrm{X}$ direction (Figure 1b). Prescribing a periodic boundary condition along the Y direction introduced a second GB interface at the end of the simulation setup, but the influence of this interface on the results was minimized by prescribing sufficient separation between the two GB planes ( $25 \mathrm{~nm})$. The dimensions along the $\mathrm{X}$ and $\mathrm{Z}$ directions were chosen to provide sufficient convergence of the 3D interfacial fracture behavior. The atomistic model was equilibrated at $300 \mathrm{~K}$ using a canonical ensemble (NVT) for $5 \mathrm{~ns}$ and the periodic directions were subsequently relaxed using the isothermal-isobaric (NPT) equations of motions for $10 \mathrm{~ns}$. Then, a through-thickness crack with a $2 \mathrm{~nm}$ height was introduced along the GB plane by deleting atoms within the crack region as illustrated in Figure $1 \mathrm{~b}$, such that the $2 a / W$ ratio for the atomistic model was kept constant at 0.1 , where $2 a$ is the crack length, and $W$ is the total width of the specimen (X dimension, $~ 50$ $\mathrm{nm})$. The minimum dimensions for the entire bicrystal at equilibrium were approximately $50 \mathrm{~nm}$ $\times 50 \mathrm{~nm} \times 5 \mathrm{~nm}(\sim 1.0$ million atoms $)$. The overall dimensions, crack size, and the crack profile used with the $\mathrm{M}(\mathrm{T})$ geometry is similar to that of what has been reported in literature for various other studies, such as $[3,15,69-71]$. The model was deformed under tensile loading by applying a constant strain rate of $10^{8} \mathrm{~s}^{-1}$ normal to the GB plane (Y direction), while the transverse boundaries were controlled using the NVT ensemble equations of motion for a temperature of $300 \mathrm{~K}$. The NVT boundary conditions produce a tensile stress in the transverse direction, which can affect the interface strength [72]. However, this lateral NVT boundary condition was deemed 
more appropriate than a stress-free NPT ensemble, as an NPT boundary condition relaxes the transverse stresses and inhibits crack propagation. The lateral stresses were on the order of $1 / 7$ th to $1 / 10$ th of the applied stress in the $\mathrm{Y}$ direction. Each bicrystal interface was deformed up to an engineering strain of $8 \%$.

The virial definition of stress was used to quantify macroscopic responses of the interface [73]. The evolution of the defected atoms (e.g., the crack tip, dislocations, and twins) was visualized using the centrosymmetry parameter [74]. This method accurately identifies defects within the crystal structure and is able to separate these defects from regions of large elastic deformation, while retaining the symmetry of their local environment during elastic deformation. The crack tip propagation was tracked using the centrosymmetry parameter; a cutoff of the centrosymmetry parameter $(>20)$ was used to identify surface atoms and track the crack trajectory.

\section{Results and Discussion}

\subsection{GB structure and energy}

Understanding the structure and energy of the GB system is crucial for engineering materials intended for advanced applications because GB properties can vary widely (coherent twin versus low angle versus high angle GBs). In this study, a range of GB structures and energies that are representative of some of the variation observed in the GB character distribution of polycrystalline as well as nanocrystalline metals was used to investigate the role of GB character on plastic events such as dislocation emission, twin formation, and atomic displacements at the interface with a preexisting crack, see Table 1.

The SUs associated with each investigated $<110>$ STGB are shown in Figure 2 with the help of Voronoi atomic volume and summarized in Table 1, along with the $0 \mathrm{~K}$ GB energy, the free volume, and the SU periodic length of each interface. The orientation of available in-plane slip systems with respect to the GB plane correlate the amount of free volume (Voronoi atomic volume) available at the interface and degree of atomic rearrangement required for heterogeneous dislocation nucleation along slip planes (Figure 2). The GB SU descriptions are in good agreement with previous descriptions available in literature $[23,28,47]$. The $\Sigma 3(111)$ coherent twin boundary with the 'D' SU (Shockley partial dislocation) along the GB plane, that exhibited small variation in the Voronoi volume at the interface. The $\Sigma 11$ (113), and $\Sigma 33$ (225) 
GB's exhibit a smaller excess atomic volume peak at the GB. This is primarily due presence of diamond shaped ' $\mathrm{C}$ ' SU that consists of 4 atoms forming a relatively tightly packed interface. The 'E' SU is made up of 6 atoms arranged in a kite shape. On the other hand, the $\Sigma 27$ (552) GB exhibits a large variation in the atomic volume at the interface, implying that the initial GB interface has a large available excess volume to accommodate deformation. The presence of large pockets of excess volume available in the GBs containing ' $E$ ' SUs at the termination of $\{111\}$ planes explains ease in a nucleating ISF that in turn lead to the dislocation nucleation [75]. These pockets of excess volume along the interface also promote cleavage behavior along the interface in the absence of dislocation nucleation ahead of the crack tip.

\subsection{Dynamic fracture using $M(T)$ for $<110>$ STGBs}

Dynamic fracture of the Al GBs was investigated with atomistic simulations of middle tension $\mathrm{M}(\mathrm{T})$ specimens at $300 \mathrm{~K}$ and a constant tensile strain rate normal to the GB plane. The stress-strain response and its correlation to the GB structures are discussed. This is followed by quantifying directional crack tip responses by measuring the crack growth with applied strains.

\subsubsection{Mechanical behavior}

The mechanical response under tension of $<110>$ STGBs as a function of applied normal strain is shown in Figure 3a. The mechanical behavior can be grouped into two distinct sets: one set for GBs with a structural period containing the ' $\mathrm{E}$ ' SU and another for GBs without the ' $\mathrm{E}$ ' SU. The presence of the 'E' SU lowers the dislocation nucleation stress, in agreement with other studies $[23,47]$. The maximum stress for all of the $<110>$ STGBs evaluated was between 1.62 GPa ( $\Sigma 9(221))$ and $2.70 \mathrm{GPa}(\Sigma 11(113))$, showing the strong effect of the GB structure. Qualitatively, the average maximum normal interface strength for GBs containing the ' $\mathrm{E}$ ' SU is about $1.63 \mathrm{GPa}$ as compared to other non-'E' SU GBs which is about $2.52 \mathrm{GPa}$. This clearly emphasizes the influence of GB structural unit on crack tip deformation, as the similar maximum stress for GBs with the 'E' SU suggest that GB dislocations drive the incipient plastic activity.

The GBs containing the 'E' SU show a lower stress response than GBs with other SUs. Figure $3 \mathrm{~b}$ shows various normalized constitutive parameters, such as GB energy and maximum normal stress as a function of misorientation of $<110>$ STGBs under tension. The GB energy and the maximum normal interface strength were normalized with $474 \mathrm{~mJ} / \mathrm{m}^{2}$ and $2.7 \mathrm{GPa}$, 
respectively. Our results indicate no particular trend for the $<110>$ STGBs under tension when comparing various interface constitutive parameters, i.e., maximum normal interface strength, GB energy, etc., except presence of ' $E$ ' $S U$ causes a large drop, on average $35 \%$, in the maximum normal interface strength for $<110>$ STGBs (see Figure $3 b$ ).

\subsubsection{Directional crack growth dynamics and the influence of GB structural units}

The crack growth along both $-\mathrm{X}[\bar{n} \bar{n} m]$ and $+\mathrm{X}[n n \bar{m}]$ direction was negligible for many interfaces that contained the 'E' SU in their structural period description (see Figure 4). All the interfaces with the ' $E$ ' $S U$ in their structural period description experienced the following sequence of deformation behavior at both crack tips, which resulted in negligible crack growth (blunting). First, with an increase in the applied strain, atomic displacements along the GB interface led to the formation of an ISF, followed by nucleation of a leading partial dislocation. The leading partial was soon followed by a twin partial dislocation along the adjacent slip plane to form the twin embryo, and then further application of strain leads to twin growth. Interestingly, for certain grain boundaries (i.e., especially in the $\Sigma 33$ (441) GB), the crack growth along both $+\mathrm{X}$ and $-\mathrm{X}$ directions was negative for long periods during the deformation process, suggesting that the crack surfaces were brought together in an attempt to minimize the energy of the system. The observed behavior ahead of the crack tip was attributed to the nucleation of multiple partial dislocations from GB sources at finite distances (10-20 А) ahead of the crack tip [76,77], which subsequently resulted in deformation twins. The analysis of the atomic mechanisms responsible for this behavior is further discussed in Section 3.2.3.2.

The directionally-dependent crack growth response was also observed in some grain boundaries (e.g., the $\Sigma 33$ (225) STGB) and the crack tip events were further analyzed during dynamic fracture (Figure 4). For example, the $\Sigma 33$ (225) STGB has an inactive period of $\sim 270$ ps, after which the stable crack growth rate was $\sim 30 \mathrm{~m} / \mathrm{s}$ and $\sim 20 \mathrm{~m} / \mathrm{s}$ along the $-\mathrm{X}[\overline{5} \overline{5} 4]$ and $+\mathrm{X}$

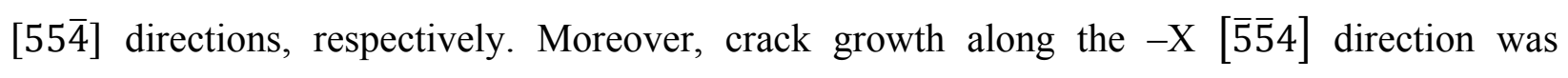
arrested from 420-575 ps due to nucleation and propagation of a partial dislocation ahead of the crack tip. In contrast, crack growth along the $+X[55 \overline{4}]$ direction did not experience a prolonged growth arrest (530-570 ps) relative to the $[\overline{5} \overline{5} 4]$ direction. The $\Sigma 33$ (225) STGB contains a high initial free volume across the interface (refer Table 1), which likely contributes to the rapid growth rate $(\sim 80 \mathrm{~m} / \mathrm{s})$ in the absence of accessible slip systems ahead of the crack tip, i.e., 
cleavage type behavior. Table 3 provides highlights of maximum periods of growth experienced and the corresponding crack tip velocity along both $-\mathrm{X}$ and $+\mathrm{X}$ directions by each interface. Interestingly, these simulations quantify that not only do different grain boundary structures and structural units influence crack growth, but they also influence the degree of anisotropy during intergranular crack growth. This anisotropy in crack growth behavior within a single grain boundary and its correlation with the atomic structure of the interface has also been observed in other studies [76-78].

\subsubsection{Analyzing deformation ahead of the crack tip}

A more detailed analysis of dynamic crack growth behavior was performed by visualizing the atomic region along the interface ahead of the mobile crack tip. The centrosymmetry parameter was used to detect dislocations and stacking faults, which have larger centrosymmetry values than atoms within a pristine fcc lattice. The two prominent deformation modes observed in the following study are highlighted below.

\subsubsection{Directional anisotropy: The role of dislocation emission}

The crack tip response was highly anisotropic for the $\Sigma 11$ (113) interface (see Figure 4), and plasticity ahead of the crack tips during fracture were studied to explain the observed mechanisms (Figure 5). At an applied strain of 2.6\% (Figure 5c), the crack along the $+\mathrm{X}[33 \overline{2}]$ direction branches out along the $\{001\}$ cleavage plane and grows in a brittle manner. On the other hand, along the $-X[\overline{3} \overline{3} 2]$ direction, steady state crack growth was observed up to an applied strain of $4.6 \%$ (Figures $4 \mathrm{a}$ ). As shown in Table 2, the relative angle between the preferred slip system and the crack plane of $100^{\circ}$ requires a large amount of energy to nucleate and propagate dislocations away from the crack tip. Coordinated atomic movement was witnessed at the interface to nucleate a partial dislocation for crack growth along the $-\mathrm{X}[\overline{3} \overline{3} 2]$ direction (Figure 5e). In contrast, minimal atomic displacements were required for the nucleation of cleavage surfaces along the $+\mathrm{X}[33 \overline{2}]$ direction. The relative orientation of slip planes with respect the crack plane and the crack growth direction was the principal cause for the crack asymmetry observed during the failure of the interface (refer Table 2 and Figure 5f). 


\subsubsection{Directional anisotropy: The role of deformation twin}

In grain boundaries with the 'E' SU, directional anisotropy of an intergranular crack during the dynamic fracture was observed. Moreover, the ' $E$ ' structural unit was identified as the cause for the twin deformation. For example, in the case of the $\Sigma 27$ (552) GB, along the $+X[11 \overline{5}]$ direction, a kink was observed in the bottom grain ahead of the crack tip which was attributed to nucleation of a microcrack along the $\{001\}$ plane followed by the nucleation of a partial dislocation and the subsequent absorption at the kink surface (Figure 6b). With an increase in the applied strain to $\sim 2 \%$, a leading partial dislocation along (111)[11ㄹ] was nucleated from the ISF with no trailing partial dislocation (Figure 6c). The extent of atomic displacement required to nucleate the partial dislocation was negligible; this behavior was consistent in all the GBs containing the 'E' SU. The leading partial was then followed by a partial dislocation on an adjacent $\{111\}$ plane, which forms a twin embryo (see Figure 6d). As the applied strain increases, successive partial (twinning) dislocations grow the twin (Figure 6e). Although twin deformation is not common in $\mathrm{Al}$, there have been several observation of twin formation and growth in Al both experimentally [12,17,21,22] and in MD simulations [2,6,16,17].

The atomic deformation ahead of the crack tip along the $-X$ [1̄15] direction is shown in Figure 6f-g. The nucleation of (111)[112] leading partial dislocation at $\varepsilon=1.7 \%$ left behind a SF in its wake, as shown in Figure 6f. This was followed by an emission of the twin

partial and growth of a twin (Figure 6g). The analysis of the deformation behavior ahead of the crack tip along both $[11 \overline{5}]$ and [ $[\overline{1} \overline{1} 5]$ directions shows that relatively minimal applied strain was required to cause the 'E' SU to rearrange and form an ISF along the interface. This ISF acted both as an accelerant in the plastic events at the interface and as a source for nucleating partial dislocations and, subsequently, deformation twins. Also, the discrete nature of these plastic events were significantly affected by the combination of availability of easy slip planes (Figure $6 \mathrm{~h}$ ), the free volume, and the SUs.

To further address the role of the 'E' SU, the key events during the deformation of $\Sigma 27$ (552) GB along both $[11 \overline{5}]$ and [1 $\overline{1} 5]$ directions of the $\mathrm{M}(\mathrm{T})$ crack were correlated with the mechanical response of the interface, as shown in Figure 7. This figure reveals some key trends which can be described as follows. As the applied normal strain increased 1) the nucleation of twin partial dislocation occurred along the $-\mathrm{X}[\overline{1} \overline{1} 5]$ of the crack as indicated in step $1(\varepsilon=$ 
$1.8 \%)$; 2) then the nucleation of twin partial dislocation along the $+\mathrm{X}[11 \overline{5}]$ direction occurred as depicted in step $2(\varepsilon=2.3 \%) ; 3)$ during segments $2-3$, twin growth occurred; 4) subsequently at step $3(\varepsilon=2.9 \%)$, another twin dislocation nucleated along $+X[11 \overline{5}]$ direction; 5) during segments 3-4, deformation twins grew; 6) after step $4(\varepsilon=3.4 \%)$, the twin partial dislocation was nucleated along the $-\mathrm{X}[\overline{1} \overline{1} 5]$ direction ahead of the crack tip; and 7) finally, at step 5 ( $\varepsilon=$ $3.7 \%$ ), nucleation of partial dislocations occurred along both [ $[\overline{1} \overline{1} 5]$ and $+X[11 \overline{5}]$ directions.

In summary, the observed incipient crack tip plastic event (Table 4) was compared against theoretically predicted behavior (Table 2). The comparison clearly shows that the observed behavior was different from the predicted behavior during dyamic fracture of the GB interface. However, the observed plastic events at the crack tip for the $\Sigma 11$ (113) GB are in agreement with previous MD studies [76] and experimental work [49]. The majority of GBs show dislocation nucleation from GB sources at a distance ahead of the crack tip (Table 4), in agreement with other studies [76,77]. This raises questions about the applicability of the Rice criterion, which suggest a dislocation emission from the crack tip. In future, we will further investigate this discrepant view through atomistic modeling of a semi-infinite crack with boundary conditions corresponding to the displacement field associated with a specified applied $\mathrm{K}$ at infinity. Overall, the significance of the present research is that it provides a physical basis for the role of GB character and crystallographic orientation on intergranular crack tip deformation behavior.

\section{Conclusions}

In this work, we use atomistic simulations to explore the role that GB structure and the adjacent crystallographic orientations have on the directional asymmetry of an intergranular crack (i.e. cleavage behavior is favored along one direction, while ductile behavior along the other direction of the interface) for aluminum grain boundaries. Simulation results from seven $<110>$ symmetric tilt grain boundaries (STGBs) show that the GB structure and the associated free volume directly influence the stress-strain response, crack growth rate, and the incipient plastic events ahead of the crack tip. In particularly, following conclusions can be drawn from the present study: 
1. The normal stress response for the $<110>$ GBs during dynamic fracture, we observed two distinct responses $\left(\theta<109.47^{\circ}\right.$ and $\left.\theta>109.47^{\circ}\right)$ of interface strength and the GB misorientation. This clearly underlines the influence of GB structural unit on the crack tip deformation, as the GBs with the ' $\mathrm{E}$ ' $\mathrm{SU}$ have similar maximum strength suggesting that the GB dislocations were driving the incipient plastic activity ahead of the crack tip.

2. To further understand this behavior, the crack growth response was studied using the $M(T)$ specimen. The crack growth for a majority of the GB interfaces studied ( $\Sigma 9$ (221), $\Sigma 11$ (332) and $\Sigma 33$ (441) GBs, containing the 'E' SU) was negligible along both directions, i.e., displaying a lack of directional anisotropy during intergranular fracture as predicted by Rice criterion. The maximum growth rate witnessed by the GB interface was in direct correlation with the initial free volume of the GB interface (Tables 1 and 2), implying that GBs with higher free volume experienced greater growth rates during cleavage deformation.

3. The 'E' SU had a significant role on the interface crack dynamics and deformation behavior when compared with other SUs. For example, we observed twin deformation only in the GB interfaces that contained 'E' SUs in GB structural description. Experimentally, similar twin formation and growth behavior has been reported in nanocrystalline $\mathrm{Al}[12,17,21,22]$.

4. Dislocation nucleation occurred from GB sites at some distance ahead of the crack tip for some GBs ( $\Sigma 9$ (221), $\Sigma 11$ (332) and $\Sigma 33$ (441)), in agreement with the findings of Cheng et al. [76] (with shaper edge crack specimens). The observed incipient crack tip plasticity for different crack sizes with these GBs question the applicability of the Rice criterion for predicting behavior of all interfaces.

5. Lastly, the GB structural unit plays a large part in dislocation nucleation ahead of the crack tip, as interface atoms provide varying degrees of mobility to incorporate plastic deformations. These new atomistic findings provide a physical basis for recognizing the role of the GB character and the boundary conditions on intergranular crack behavior.

\section{Acknowledgement}

The authors would like to recognize Dr. W. Mullins and Dr. A.K. Vasudevan from the Office of Naval Research for providing insights and valuable suggestions. This material is based upon work supported by the Office of Naval Research under contract no. N000141110793. We would also like to acknowledge the Fulton High Performance Computing at Arizona State University. 


\section{References}

[1] S. Yip, D. Wolf, in:, Mater. Sci. Forum, 1991, pp. 77-168.

[2] D. Farkas, M. Duranduru, W.A. Curtin, C. Ribbens, Philos. Mag. A 81 (2001) 1241.

[3] S. Hai, E. Tadmor, Acta Mater. 51 (2003) 117.

[4] H. Li, N. Chandra, Int. J. Plast. 19 (2003) 849.

[5] T. Zhu, J. Li, S. Yip, Phys. Rev. Lett. 93 (2004) 025503.

[6] Y.T. Zhu, X.Z. Liao, S.G. Srinivasan, Y.H. Zhao, M.I. Baskes, F. Zhou, E.J. Lavernia, Appl. Phys. Lett. 85 (2004) 5049.

[7] Z.J. Zhang, G.H. Paulino, Int. J. Plast. 21 (2005) 1195.

[8] M.J. Buehler, H. Gao, Nature 439 (2006) 307.

[9] V. Yamakov, E. Saether, D.R. Phillips, E.H. Glaessgen, J. Mater. Sci. 42 (2007) 1466.

[10] V.I. Yamakov, E.H. Glaessgen, Nat. Mater. 6 (2007) 795.

[11] S.V. Bobylev, A.K. Mukherjee, I.A. Ovid'ko, A.G. Sheinerman, Int. J. Plast. 26 (2010) 1629.

[12] X. Liao, F. Zhou, E. Lavernia, S. Srinivasan, M. Baskes, D. He, Y. Zhu, Appl. Phys. Lett. 83 (2003) 632.

[13] D. Warner, W. Curtin, S. Qu, Nat. Mater. 6 (2007) 876.

[14] L. Xiong, Q. Deng, G.J. Tucker, D.L. McDowell, Y. Chen, Int. J. Plast. 38 (2012) 86.

[15] E. Tadmor, S. Hai, J. Mech. Phys. Solids 51 (2003) 765.

[16] V. Yamakov, D. Wolf, S.R. Phillpot, H. Gleiter, Acta Mater. 50 (2002) 5005.

[17] B.Q. Li, M.L. Sui, B. Li, E. Ma, S.X. Mao, Phys. Rev. Lett. 102 (2009) 205504.

[18] R.C. Pond, L.M.F. Garcia-Garcia, Inst Phys Conf Ser. 61, (1982), pp. 495-498.

[19] Y.T. Zhu, X.Z. Liao, X.L. Wu, Prog. Mater. Sci. 57 (2012) 1.

[20] V. Yamakov, D. Wolf, S. Phillpot, A. Mukherjee, H. Gleiter, Nat. Mater. 3 (2003) 43.

[21] M. Chen, E. Ma, K.J. Hemker, H. Sheng, Y. Wang, X. Cheng, Science 300 (2003) 1275.

[22] D. Wolf, V. Yamakov, S.R. Phillpot, A. Mukherjee, H. Gleiter, Acta Mater. 53 (2005) 1.

[23] D.E. Spearot, K.I. Jacob, D.L. McDowell, Int. J. Plast. 23 (2007) 143.

[24] M.A. Tschopp, D.L. McDowell, Int. J. Plast. 24 (2008) 191.

[25] A. Sutton, V. Vitek, Philos. Trans. R. Soc. LondonSeries Math. Phys. Sci. 309 (1983) 1.

[26] G.J. Wang, A. Sutton, V. Vitek, Acta Metall. 32 (1984) 1093.

[27] W. Gui-Jin, V. Vitek, Acta Metall. 34 (1986) 951.

[28] J. Rittner, D. Seidman, Phys. Rev. B 54 (1996) 6999.

[29] M. Rajagopalan, M.A. Bhatia, M.A. Tschopp, D.J. Srolovitz, K.N. Solanki, Acta Mater. 73 (2014) 312.

[30] K.N. Solanki, M.A. Tschopp, M.A. Bhatia, N.R. Rhodes, Metall. Mater. Trans. A 44 (2013) 1365.

[31] J.M. Burgers, Proc. Phys. Soc. 52 (1940) 23.

[32] L. Bragg, J.F. Nye, Proc. R. Soc. Lond. Ser. Math. Phys. Sci. (1947) 474.

[33] W.T. Read, W. Shockley, Phys. Rev. 78 (1950) 275.

[34] G.H. Bishop, B. Chalmers, Scr. Metall. 2 (1968) 133.

[35] J.. Hirth, Acta Metall. 22 (1974) 1023.

[36] H. Grimmer, W. Bollmann, D.H. Warrington, Acta Crystallogr. A 30 (1974) 197.

[37] M.A. Bhatia, K.N. Solanki, J. Appl. Phys. 114 (2013) 244309.

[38] M. Rajagopalan, M.A. Tschopp, K.N. Solanki, JOM 66 (2014) 129. 
[39] N.R. Rhodes, M.A. Tschopp, K.N. Solanki, Model. Simul. Mater. Sci. Eng. 21 (2013) 035009 .

[40] P.D. Bristowe, A.G. Crocker, Philos. Mag. A 38 (1978) 487.

[41] R.C. Pond, Philos. Mag. A 39 (1979) 679.

[42] R.C. Pond, D.A. Smith, V. Vitek, Acta Metall. 27 (1979) 235.

[43] K.W. Ingle, A.G. Crocker, Philos. Mag. A 41 (1980) 713.

[44] V. Vitek, D. Smith, R. Pond, Philos. Mag. A 41 (1980) 649.

[45] A. Sutton, Philos. Mag. A 46 (1982) 171.

[46] A. Sutton, Philos. Mag. Lett. 59 (1989) 53.

[47] F. Sansoz, J.F. Molinari, Acta Mater. 53 (2005) 1931.

[48] D.E. Spearot, K.I. Jacob, D.L. McDowell, Acta Mater. 53 (2005) 3579.

[49] J.-S. Wang, P.M. Anderson, Acta Metall. Mater. 39 (1991) 779.

[50] M. Saeedvafa, Mech. Mater. 13 (1992) 295.

[51] J.-S. Wang, S.D. Mesarovic, Acta Metall. Mater. 43 (1995) 3837.

[52] J.W. Kysar, Acta Mater. 48 (2000) 3509.

[53] J.W. Kysar, J. Mech. Phys. Solids 49 (2001) 1099.

[54] H. Tang, A. Acharya, S. Saigal, Mech. Mater. 37 (2005) 593.

[55] J.R. Rice, J. Mech. Phys. Solids 40 (1992) 239.

[56] D.A. Smith, V. Vitek, R.C. Pond, Acta Metall. 25 (1977) 475.

[57] A. Sutton, V. Vitek, Philos. Trans. R. Soc. LondonSeries Math. Phys. Sci. 309 (1983) 37.

[58] V. Vitek, A. Sutton, G.J. Wang, D. Schwartz, Scr. Metall. 17 (1983) 183.

[59] S. Cheng, E. Ma, Y. Wang, L. Kecskes, K. Youssef, C. Koch, U. Trociewitz, K. Han, Acta Mater. 53 (2005) 1521.

[60] S. Plimpton, J. Comput. Phys. 117 (1995) 1.

[61] M.S. Daw, M.I. Baskes, Phys. Rev. B 29 (1984) 6443.

[62] X.Y. Liu, F. Ercolessi, J.B. Adams, Model. Simul. Mater. Sci. Eng. 12 (2004) 665.

[63] Y. Mishin, D. Farkas, M.J. Mehl, D.A. Papaconstantopoulos, Phys. Rev. B 59 (1999) 3393.

[64] M.J. Buehler, Atomistic Modeling of Materials Failure, Springer, New York, 2008.

[65] M.J. Mills, M.S. Daw, G.J. Thomas, F. Cosandey, Ultramicroscopy 40 (1992) 247.

[66] D.L. Medlin, M.J. Mills, W.M. Stobbs, M.S. Daw, F. Cosandey, in:, MRS Proc., 1992, p. 91.

[67] P. Grigoriadis, T. Karakostas, P. Komninou, V. Pontikis, in:, Mater. Sci. Forum, 1998, pp. $177-180$.

[68] S.P. Chen, D.J. Srolovitz, A.F. Voter, J Mater Res 4 (1989) 62.

[69] J. Song, W.A. Curtin, Nat. Mater. (2012).

[70] G.Q. Xu, M.J. Demkowicz, Phys. Rev. Lett. 111 (2013) 145501.

[71] J. Zhang, S. Ghosh, J. Mech. Phys. Solids 61 (2013) 1670.

[72] T. Kitamura, K. Yashiro, R. Ohtani, JSME Int. J. Ser. A 40 (1997) 430.

[73] D.H. Tsai, J. Chem. Phys. 70 (1979) 1375.

[74] C.L. Kelchner, S.J. Plimpton, J.C. Hamilton, Phys. Rev. B 58 (1998) 11085.

[75] M.A. Tschopp, G.J. Tucker, D.L. McDowell, Acta Mater. 55 (2007) 3959.

[76] Y. Cheng, Z.-H. Jin, Y.W. Zhang, H. Gao, Acta Mater. 58 (2010) 2293.

[77] Y. Cheng, M.X. Shi, Y.W. Zhang, Int. J. Solids Struct. 49 (2012) 3345.

[78] V. Yamakov, E. Saether, D.R. Phillips, E.H. Glaessgen, Phys. Rev. Lett. 95 (2005) 15502. 


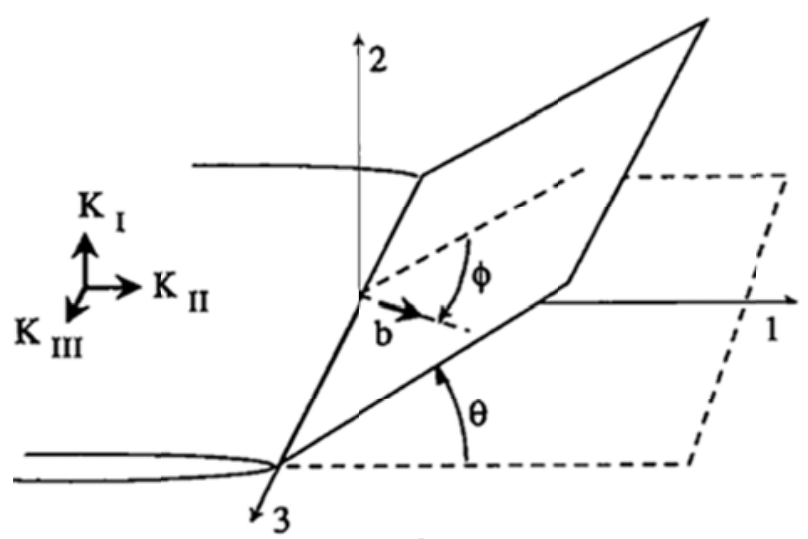

a)

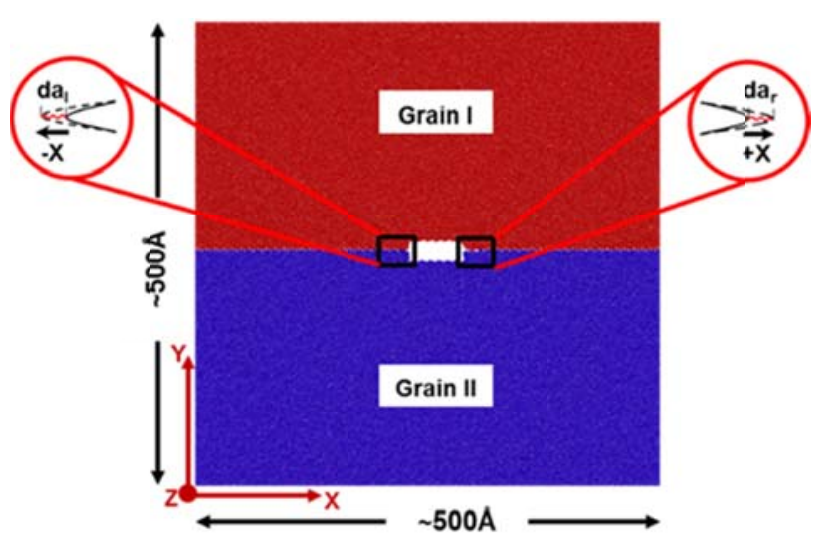

b)

Figure 1. a) A schematic representation of geometric variables $\theta$ and $\phi$ in the Rice criterion, adapted from [55]. The slip plane inclined at an angle $\theta$, and the crack plane and slip direction inclined at an angle $\phi$ with the crack growth direction b) Atomistic cell with a GB interface perpendicular to the $\mathrm{Y}$ axis. The red and blue regions are grains I and II, respectively. For grains I and II, the $\mathrm{Z}$ tilt axis is the $<110>$ orientation. Tensile load was applied along the $\mathrm{Y}$ axis to investigate GB SUs' role on plastic events and any subsequent interfacial failure behavior. 


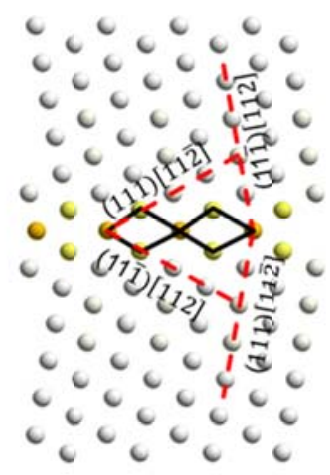

(a) $\Sigma 11(113) 50.48^{\circ}$ |C.C|

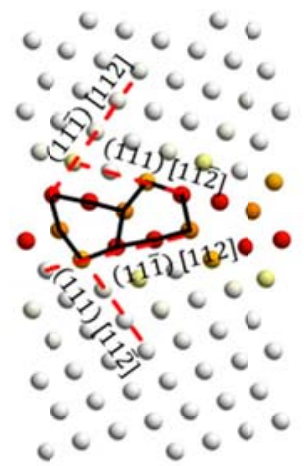

(e) $\Sigma 9$ (221) $141.06^{\circ}$

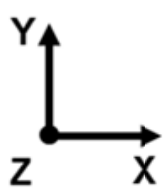

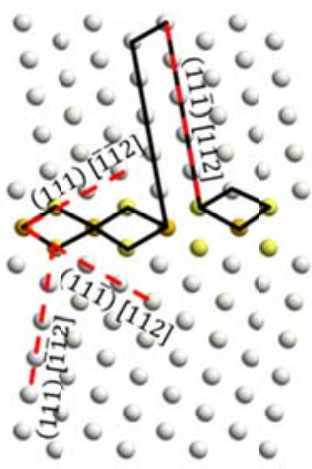

(b) $\Sigma 33(225) 59^{\circ}$ |C.CDC.C|

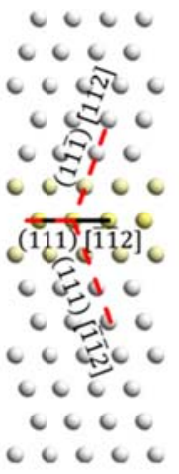

(c) $\Sigma 3$ (111) $109.48^{\circ}$

|D.D|

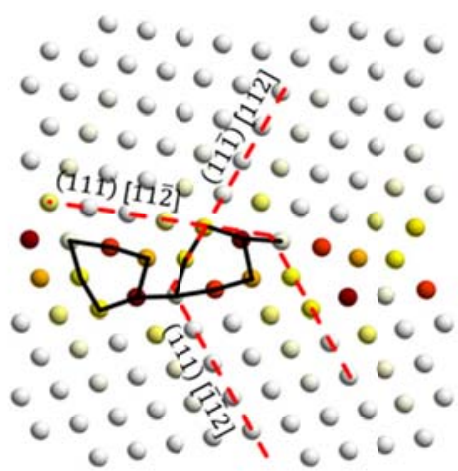

(d) $\Sigma 11(332) 129.52^{\circ}$

|DE.DE|

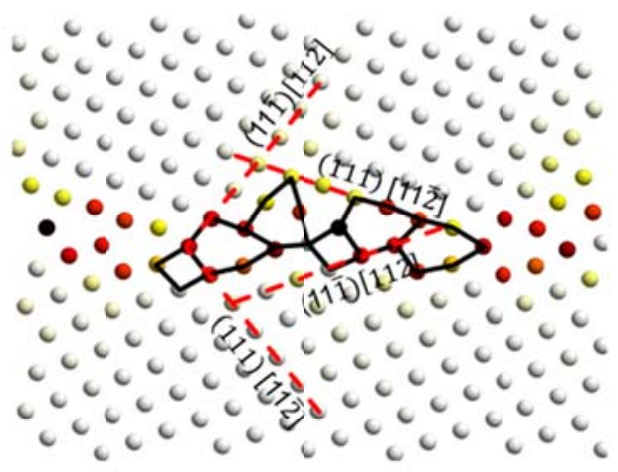

(f) $\Sigma 27$ (552) $148.42^{\circ}$ |AEE.AEE|

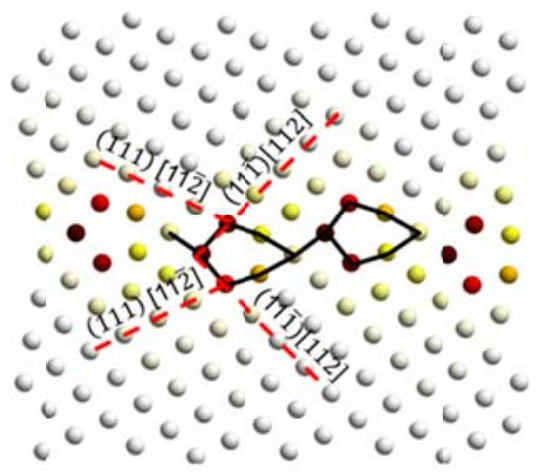

(g) $\Sigma 33$ (441) $159.96^{\circ}$ |ED.ED|

\section{$V_{\text {voro }}$}

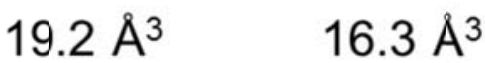

Figure 2. $<110>$ symmetric tilt GB structures with structural units outlined for a) $\Sigma 11$ (113) $\left(\theta=50.48^{\circ}\right)$, b) $\Sigma 33(225)\left(\theta=59^{\circ}\right)$, c) $\Sigma 3(111)\left(\theta=109.47^{\circ}\right)$, d) $\Sigma 11$ (332) $\left(\theta=129.52^{\circ}\right)$, e) $\Sigma 9$ (221) $\left(\theta=141.06^{\circ}\right)$, f) $\Sigma 27(552)\left(\theta=148.52^{\circ}\right)$ and g) $\Sigma 33(441)\left(\theta=159.96^{\circ}\right)$. Atoms are colored based on Voronoi volume on a scale of $19.2 \AA^{3}$ to $16.3 \AA^{3}$. The favorable slip planes (111) and (111) are denoted using dotted lines. This helps in visualizing the initial orientation of slip planes with respect to the GB structure and the amount of excess volume available to the GB atoms in order to nucleate a dislocation along these slip planes. 


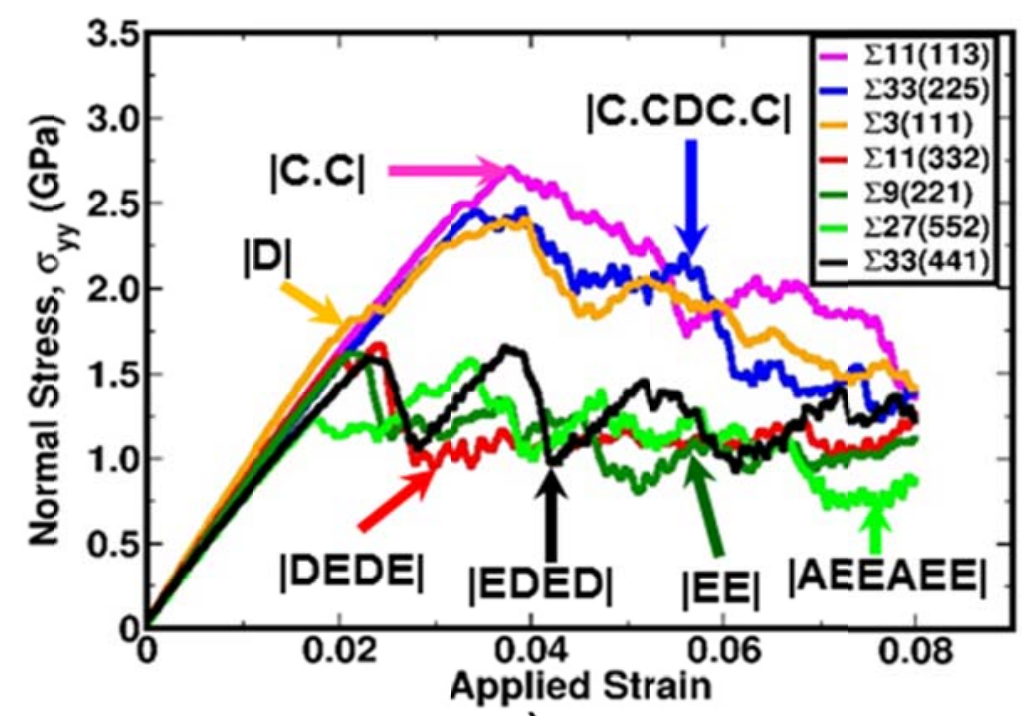

a)

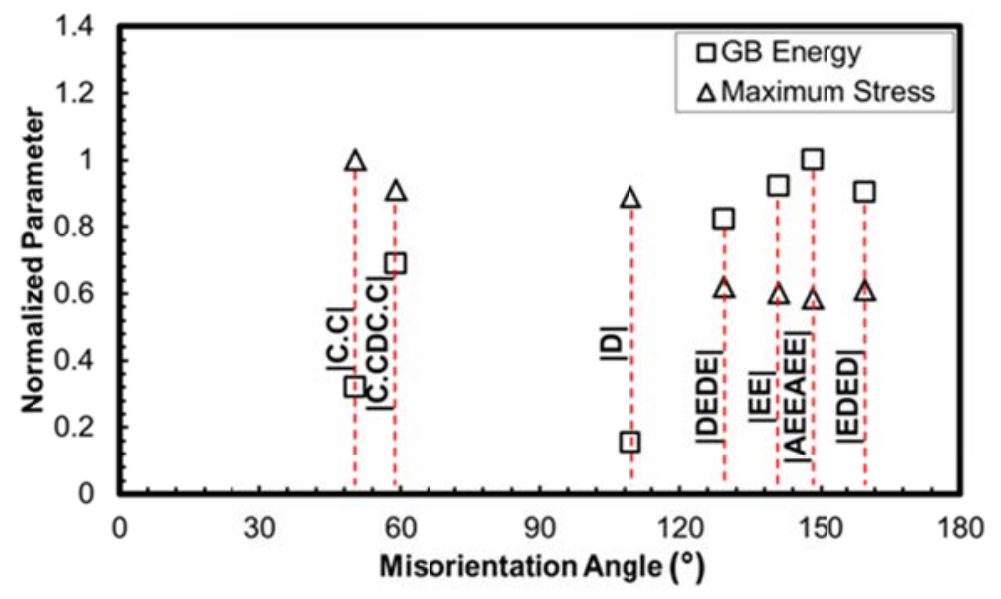

b)

Figure 3. Stress-strain behavior of various $<110>$ GB interfaces under tensile loading. The simulation results indicate influence of SU on the maximum normal stress encountered by the GB interface, especially the 'E' SU, and b) various normalized constitutive parameters of $<110>$ STGBs under tension. The GB energy and the maximum normal stress were normalized with $474 \mathrm{~mJ} / \mathrm{m}^{2}$ and $2.7 \mathrm{GPa}$, respectively. 


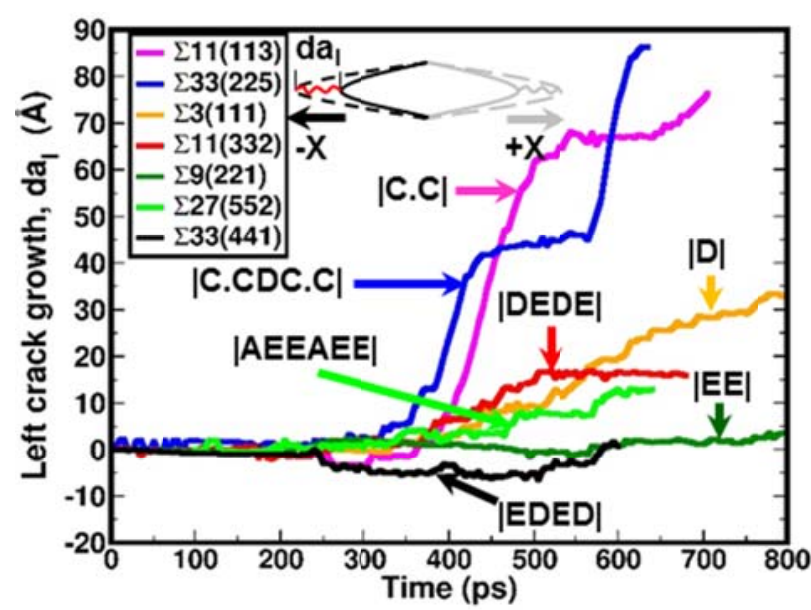

a)

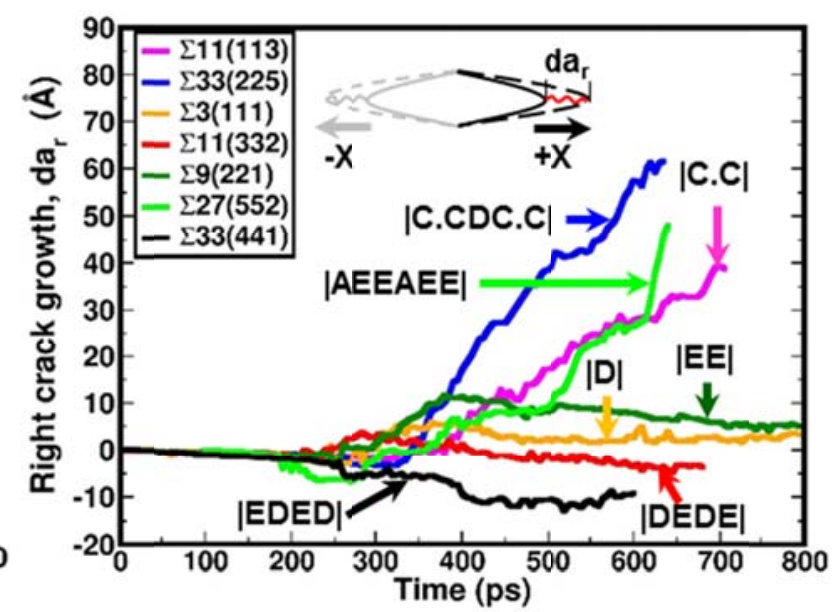

b)

Figure 4. The crack growth evolution in various $<110>$ GBs exhibits distinct features, such as inactive, continuous growth, arrest, second continuous growth, followed by final failure: a) the left $(-\mathrm{X}[\bar{n} \bar{n} m]$ direction), and $\mathrm{b})$ the right $(+\mathrm{X}[n n \bar{m}]$ direction) sides of the middle tension specimen. Note that the equivalent applied strain for an elapsed time of 1 ps is $10^{-4}$ (i.e., the applied strain rate of $10^{8}$ ). 

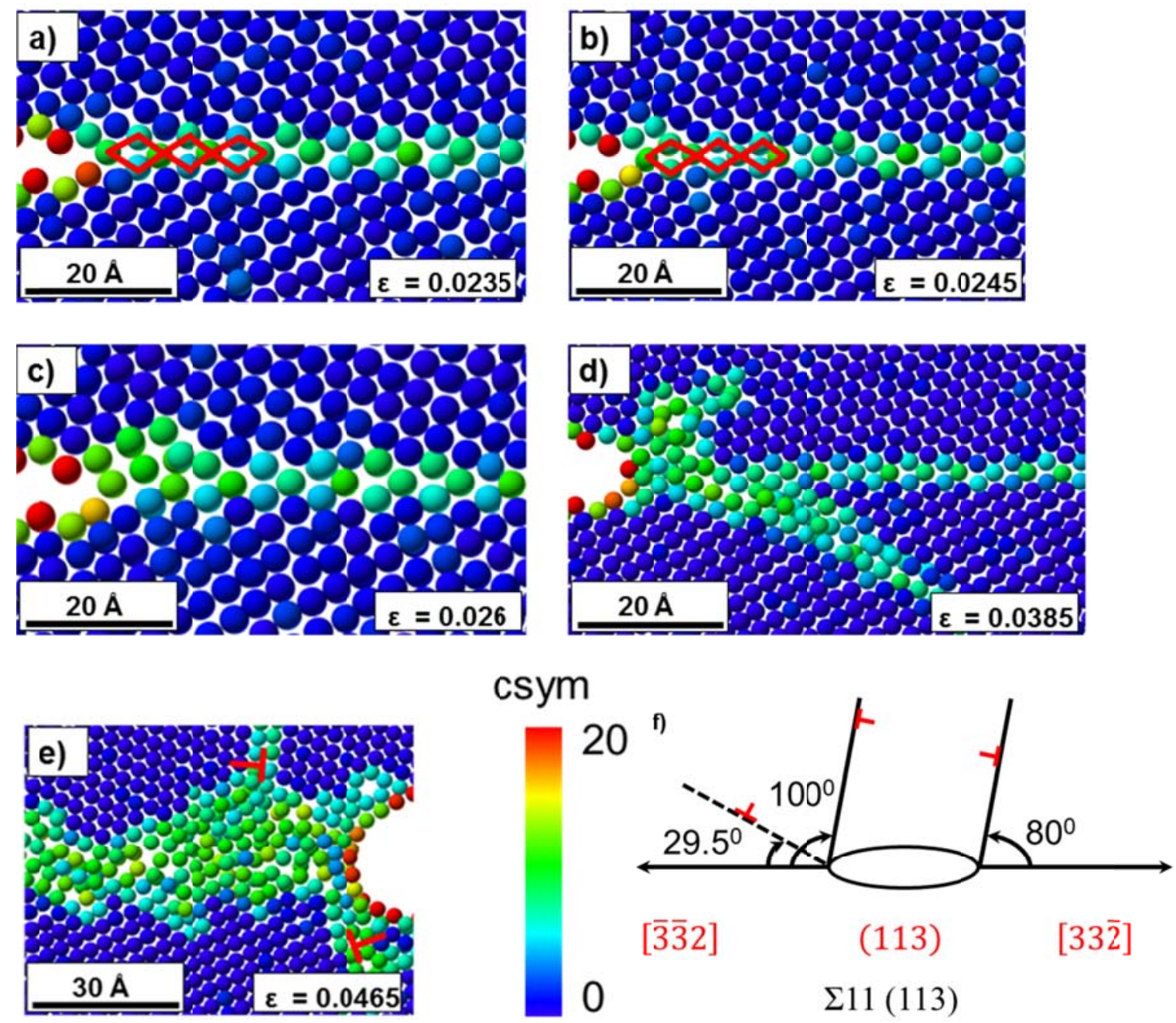

Figure 5. The plastic events across the $\Sigma 11$ (113) GB interface with atoms were identified using the centrosymmetry parameter (csym). (a-d) show plastic events during the crack growth along the $+\mathrm{X}$ direction; (e) illustrates the dislocation nucleation during the crack propagation along the $-\mathrm{X}$ direction. f) an illustration of the preferred slip system orientation with respect to the GB plane for the $\Sigma 9$ (221) GB during crack propagation along both directions. The dashed line depicts the orientation of the second favored slip system. Note that the equivalent applied strain for an elapsed time of $1 \mathrm{ps}$ is $10^{-4}$ (i.e., the applied strain rate of $10^{8}$ ). 

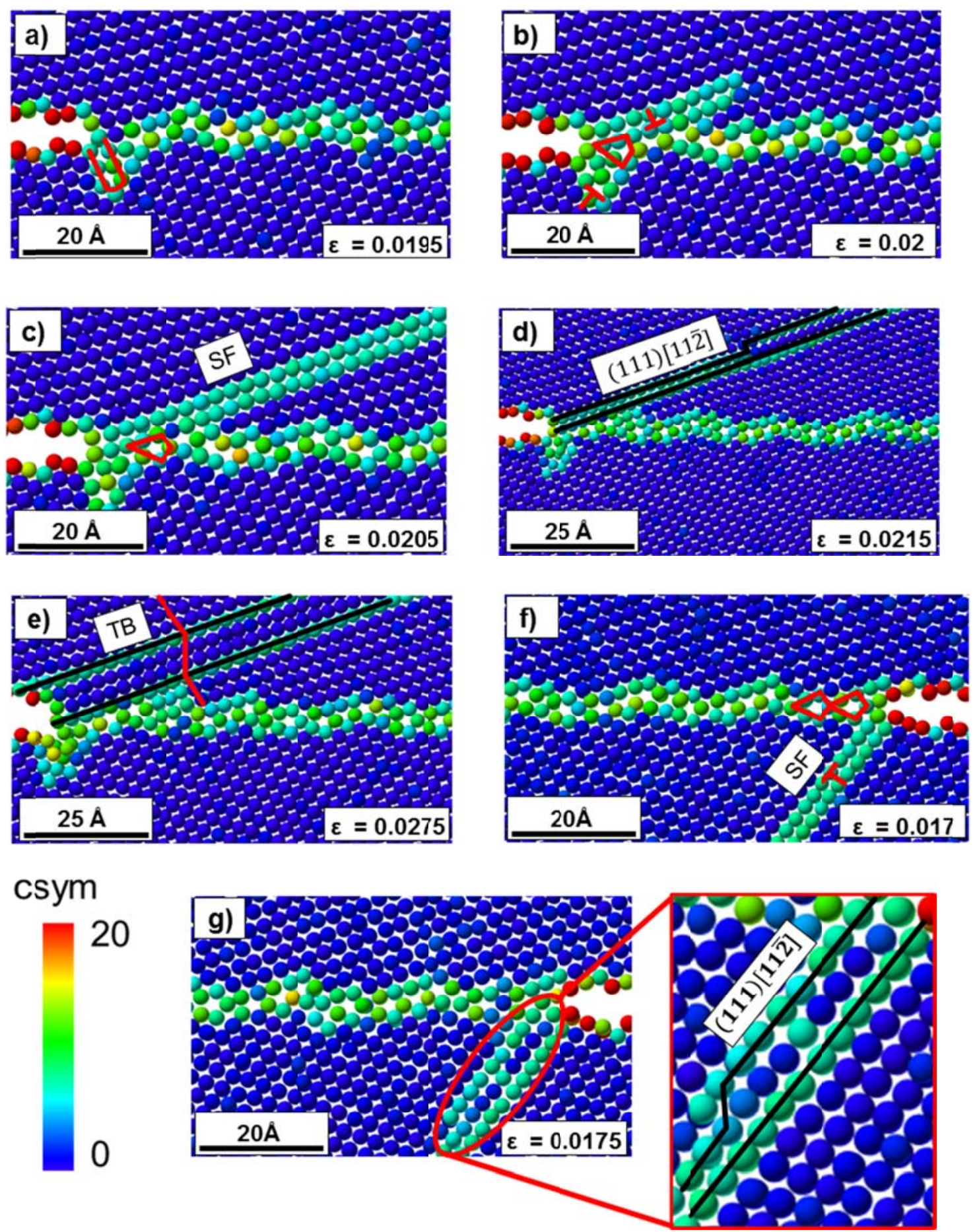


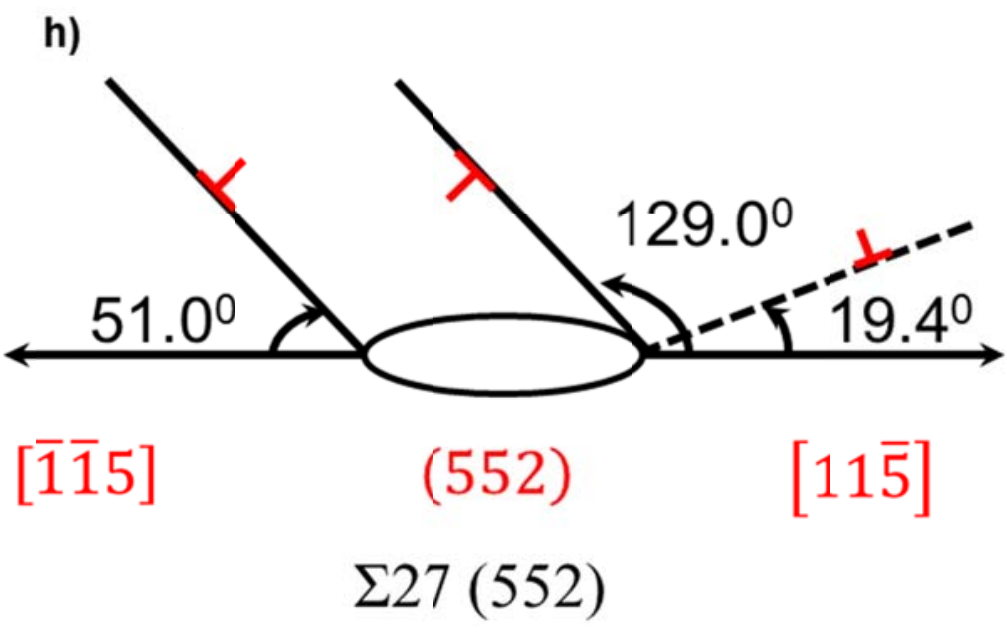

Figure 6. The plastic events across the $\Sigma 27$ (552) GB interface with atoms are identified using centrosymmetry (csym). (a-e) Show a nucleation of twin followed by growth of the crack tip along the $+\mathrm{X}$ direction, and (f-g) illustrate the twin nucleation and growth of the crack tip along the $-\mathrm{X}$ direction. The insets magnify the area of interest depicting the twin formation. $\mathrm{h}$ ) an illustration of the preferred slip system orientation with respect to the GB plane for $\Sigma 27$ (552) GB during crack propagation along both directions. The dashed line depicts the orientation of the second favored slip system. The equivalent applied strain for an elapsed time of $1 \mathrm{ps} \mathrm{is} 10^{-4}$ (i.e. applied strain rate of $10^{8}$ ).

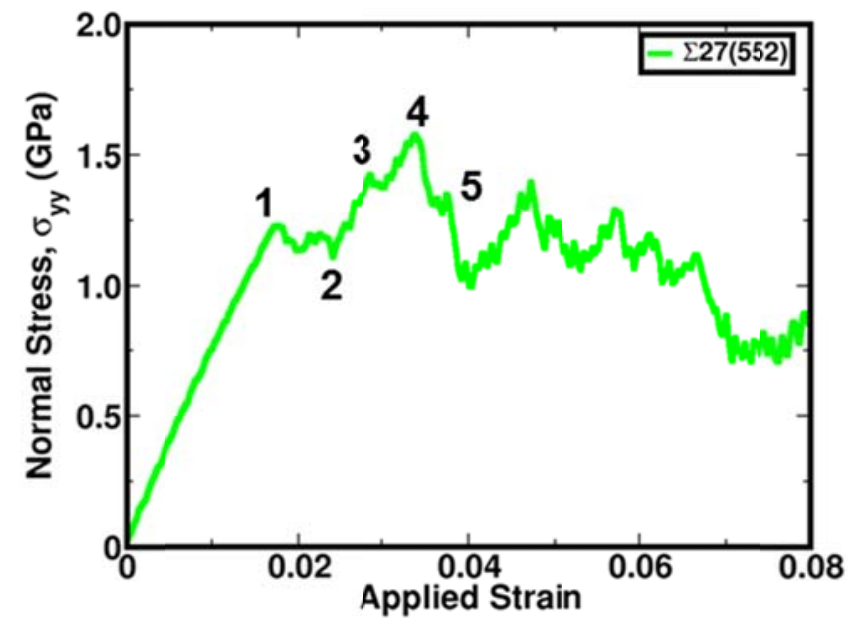

Figure 7. Mechanical response and highlights of key events (1-5) taking place during the deformation of $\Sigma 27$ (552) $\theta=31.58^{\circ} \mathrm{GB}$ interface. Note: $10^{-2}$ applied strain $=100 \mathrm{ps}$. 
Table 1. Grain boundary CSL description, SU notation, the GB periodic length, GB energy, the free volume used in this work. The $\Sigma 27$ (552) GB has the highest initial free volume, periodic length and GB energy among $<110>$ STGBs examined here.

\begin{tabular}{cccccc}
\hline $\begin{array}{c}\text { Misorientation } \\
\text { angle } \\
(\boldsymbol{\theta})\end{array}$ & $\begin{array}{c}\text { CSL } \\
\text { designation } \\
(\boldsymbol{\Sigma})\end{array}$ & $\begin{array}{c}\text { SUM } \\
\text { notation }\end{array}$ & $\begin{array}{c}\text { GB periodic } \\
\text { length }(\mathbf{\AA})\end{array}$ & $\begin{array}{c}\text { GB } \\
\text { energy } \\
\left(\mathbf{m J} / \mathbf{m}^{\mathbf{2}}\right)\end{array}$ & $\begin{array}{c}\text { Free volume } \\
\left(\mathbf{\Delta \mathbf { z } ^ { * } )}\right. \\
\text { in units of } \mathbf{a}_{\mathbf{0}}\end{array}$ \\
\hline $\mathbf{5 0 . 4 8}^{\circ}$ & $\Sigma 11(113)$ & $\mid$ C.C & 18.91 & 151 & 0.1811 \\
$\mathbf{5 9 . 0 0}^{\circ}$ & $\Sigma 33(225)$ & $\mid$ C.CDC.C $\mid$ & 32.75 & 326 & 0.2060 \\
${\mathbf{1 0 9 . 4 7 ^ { \circ }}}^{\circ}$ & $\Sigma 3(111)$ & $\mid$ D.D $\mid$ & 9.87 & 73 & 0.0005 \\
$\mathbf{1 2 9 . 5 2}^{\circ}$ & $\Sigma 11(332)$ & $\mid$ DE.DE $\mid$ & 26.75 & 390 & 0.1724 \\
$\mathbf{1 4 1 . 0 6}^{\circ}$ & $\Sigma 9(221)$ & $\mid$ E.E $\mid$ & 17.10 & 437 & 0.1500 \\
$\mathbf{1 4 8 . 4 2}^{\circ}$ & $\Sigma 27(552)$ & $\mid$ AEE.AEE $\mid$ & 41.90 & 474 & 0.2650 \\
$\mathbf{1 5 9 . 9 6}^{\circ}$ & $\Sigma 33(441)$ & $\mid$ ED.ED $\mid$ & 32.75 & 428 & 0.1610 \\
\hline
\end{tabular}

Table 2. Predicted minimum energy slip plane orientation with respect to the crack plane along both $-X$ and $+X$ direction of crack growth $(\theta)$ based on the Rice model (refer equation 1 ). The energy release rate for GB cleavage $\left(\mathrm{G}_{c l e a}=2 \gamma_{s^{-}} \gamma_{g b}\right)$ and dislocation nucleation ahead of the crack tip along both directions.

\begin{tabular}{|c|c|c|c|c|c|}
\hline $\begin{array}{c}\text { Grain } \\
\text { boundary, } \\
\text { CSL } \\
\text { designation } \\
(\Sigma)\end{array}$ & $\begin{array}{c}\text { Critical } \\
\text { energy } \\
\text { release rate } \\
\text { for cleavage } \\
\left(G_{\text {clea }}\right), \mathbf{J} / \mathbf{m}^{2}\end{array}$ & $\begin{array}{c}\text { Relative angle } \\
\text { between slip } \\
\text { plane and } \\
\text { crack plane } \\
\text { along the }-\mathrm{X} \\
\text { direction }\left(\theta^{-}\right), \\
\text {degrees }\end{array}$ & $\begin{array}{c}\text { Relative angle } \\
\text { between slip } \\
\text { plane and crack } \\
\text { plane along the } \\
+X \text { direction }\left(\theta^{+}\right) \\
\text {degrees }\end{array}$ & $\begin{array}{c}\text { Critical energy } \\
\text { release rate for } \\
\text { dislocation } \\
\text { nucleation } \\
\text { along the }-\mathrm{X} \\
\text { direction } \\
\left(G_{\text {disl }}^{-}\right), \mathrm{J} / \mathbf{m}^{2} \\
\end{array}$ & $\begin{array}{c}\text { Critical energy } \\
\text { release rate for } \\
\text { dislocation } \\
\text { nucleation } \\
\text { along the }+\mathrm{X} \\
\text { direction } \\
\left(G_{d i s l}^{+}\right), \mathrm{J} / \mathbf{m}^{2} \\
\end{array}$ \\
\hline$\Sigma 11(113)$ & 1.28 & 100.0 & 80.0 & 1.28 & 0.90 \\
\hline$\Sigma 33(225)$ & 1.14 & 95.7 & 84.23 & 1.15 & 0.94 \\
\hline$\Sigma 3(111)$ & 1.23 & 109.5 & 70.5 & 1.73 & 0.86 \\
\hline$\Sigma 11(332)$ & 0.93 & 60.5 & 119.5 & 0.90 & 2.66 \\
\hline$\Sigma 9(221)$ & 0.92 & 54.7 & 125.3 & 0.97 & 3.62 \\
\hline$\Sigma 27(552)$ & 1.28 & 51.0 & 129.0 & 1.04 & 4.56 \\
\hline$\Sigma 33(441)$ & 1.11 & 45.3 & 25.2 & 1.19 & 2.96 \\
\hline
\end{tabular}


Table 3. Crack propagation figures of merit along the $+X$ and $-X$ directions for $<110>$ STGB interfaces.

\begin{tabular}{|c|c|c|c|c|c|c|}
\hline $\begin{array}{c}\text { CSL } \\
\text { designation } \\
(\Sigma)\end{array}$ & $\begin{array}{c}\text { Maximum } \\
\text { crack } \\
\text { growth } \\
\text { period along } \\
\text { the }-X \\
\text { direction } \\
\text { (ps) } \\
\end{array}$ & $\begin{array}{l}\text { Maximum } \\
\text { crack } \\
\text { growth } \\
\text { along the -X } \\
\text { direction } \\
(\AA)\end{array}$ & $\begin{array}{c}\text { Maximum } \\
\text { crack } \\
\text { growth } \\
\text { velocity } \\
\text { along the }-X \\
\text { direction } \\
(\mathrm{m} / \mathrm{s}) \\
\end{array}$ & $\begin{array}{c}\text { Maximum } \\
\text { crack } \\
\text { growth } \\
\text { period along } \\
\text { the }+X \\
\text { direction } \\
\text { (ps) } \\
\end{array}$ & $\begin{array}{l}\text { Maximum } \\
\text { crack } \\
\text { growth } \\
\text { along the } \\
+X \\
\text { direction } \\
(\AA) \\
\end{array}$ & $\begin{array}{c}\text { Maximum } \\
\text { crack } \\
\text { growth } \\
\text { velocity } \\
\text { along the }+X \\
\text { direction } \\
(\mathbf{m} / \mathbf{s}) \\
\end{array}$ \\
\hline$\Sigma 11(113)$ & $370-500$ & 76 & 48 & $375-600$ & 39 & 12 \\
\hline$\Sigma 33(225)$ & $570-615$ & 86 & 85 & $320-510$ & 62 & 22 \\
\hline 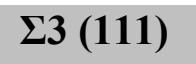 & $400-650$ & 33 & 10 & $300-325$ & 6 & 20 \\
\hline$\Sigma 11(332)$ & $360-500$ & 17 & 10 & $250-290$ & 4 & 10 \\
\hline$\Sigma 9(221)$ & NA & 3 & - & $300-380$ & 12 & 12 \\
\hline$\Sigma 27(552)$ & $570-600$ & 13 & 24 & $610-650$ & 48 & 50 \\
\hline$\Sigma 33(441)$ & NA & 2 & - & NA & 0 & - \\
\hline
\end{tabular}

Table 4. The observed crack tip events along both directions of the crack propagation for various GBs during the dynamic fracture are summarized. The dislocation (D), dislocation nucleation from GB sites ahead of the crack tip (H.D), and the cleavage (C) are the incipient crack tip events classified below.

\begin{tabular}{ccc}
\hline $\begin{array}{c}\text { Grain boundary, } \\
\text { CSL designation }(\Sigma)\end{array}$ & $\begin{array}{c}\text { Incipient plastic event } \\
\text { ahead of the crack tip } \\
\text { along the }- \text { X direction }\end{array}$ & $\begin{array}{c}\text { Incipient plastic event } \\
\text { ahead of the crack tip } \\
\text { along the +X direction }\end{array}$ \\
\hline $\boldsymbol{\Sigma} 11(\mathbf{1 1 3})$ & D & C \\
$\boldsymbol{\Sigma} 33(\mathbf{2 2 5})$ & D & C \\
$\boldsymbol{\Sigma} 3(\mathbf{1 1 1})$ & H.D & H.D \\
$\boldsymbol{\Sigma} 11(\mathbf{3 3 2})$ & H.D & H.D \\
$\boldsymbol{\Sigma} 9(\mathbf{2 2 1})$ & H.D & H.D \\
$\boldsymbol{\Sigma} 27(552)$ & D & D \\
$\boldsymbol{\Sigma} 33(\mathbf{4 4 1 )}$ & H.D & H.D \\
\hline
\end{tabular}

